\title{
Ab Initio Study of Structural, Electronic, and Hyperfine Properties of n-type $\mathrm{SnO}_{2}: \mathrm{Ta}$ Semiconductor
}

\author{
Germán N. Darriba, ${ }^{*}, \dagger$ Emiliano L. Muñoz, ${ }^{\ddagger}$ Leonardo A. Errico, ${ }^{\dagger, \ddagger \S \S}$ and Mario Rentería ${ }^{\dagger, \ddagger}$ \\ ${ }^{\dagger}$ Instituto de Física La Plata (IFLP, CONICET La Plata), Facultad de Ciencias Exactas, Universidad Nacional de La Plata, CC 67, \\ 1900 La Plata, Argentina \\ ${ }^{\ddagger}$ Departamento de Física, Facultad de Ciencias Exactas, Universidad Nacional de La Plata, CC 67, 1900, La Plata, Argentina \\ ${ }^{\S}$ Universidad Nacional del Noroeste de la Provincia de Buenos Aires (UNNOBA), Monteagudo 2772, Pergamino, C P 2700, Buenos \\ Aires, Argentina
}

\begin{abstract}
A detailed theoretical first-principles study of structural, electronic, and hyperfine properties at $\mathrm{Sn}$ and $\mathrm{Ta}$ sites of undoped and Ta-doped rutile $\mathrm{SnO}_{2}$ is presented, using the Full-Potential Augmented Plane Wave plus local orbitals (FP-APW+lo) method. In the Ta-doped systems, we performed calculations for two different charge states. The predicted electric-field-gradient (EFG) tensor, the key magnitude in this study, for both charge states of the impurity result to be almost equal and in good agreement with TimeDifferential Perturbed $\gamma-\gamma$ Angular Correlation (TDPAC) results in ${ }^{181}$ Ta-doped $\mathrm{SnO}_{2}$ thin films. This study enables at present to discuss the origin of the EFG and the role played by the structural anisotropic contractions introduced by the Ta atom and the impurity charge state on the hyperfine properties. To determine the correct charge state of the impurity, we performed energetic studies, predicting the metallic behavior of degenerate semiconductors, in agreement with resistivity experimental results obtained in samples with the same Ta dilution.
\end{abstract}

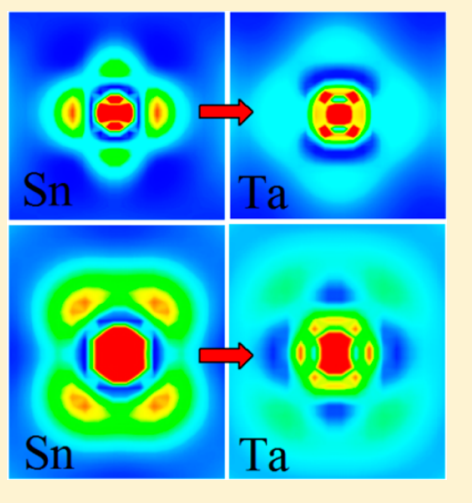

\section{INTRODUCTION}

In past years, transparent conducting oxides (TCO) thin films have been increasingly used in optoelectronic devices such as solar cells, flat-panel displays, and, more recently, touch panels. Initially, $\mathrm{In}_{2} \mathrm{O}_{3}$ doped with $\mathrm{Sn}$ as n-type donor (indium-tin oxide, ITO) was the most used TCO. However, the difficulty to obtain it and its high cost have made necessary the exploration of alternative materials. $\mathrm{ZnO}$ and $\mathrm{SnO}_{2}$ can also be heavily doped with n-type donors and are the principal candidates to replace ITO. Thin films based on $\mathrm{SnO}_{2}$, such as Sb-doped $\mathrm{SnO}_{2},{ }^{1-3}$ are the most chemically stable; however, the toxicity of $\mathrm{Sb}$ makes it undesirable. In this sense, Ta-doped rutile $\mathrm{SnO}_{2}$ thin films (TTO) have proven to be an ideal candidate. ${ }^{4-8} \mathrm{Ta}-$ doping in rutile $\mathrm{SnO}_{2}$ thin films does not produce a significant loss in their transparency ${ }^{7}$ and decreases their resistivity from $0.17 \Omega \mathrm{cm}$ (pure $\mathrm{SnO}_{2}$ film) to $1.1 \times 10^{-4} \Omega \mathrm{cm}$ $\left(\mathrm{Sn}_{0.95} \mathrm{Ta}_{0.05} \mathrm{O}_{2}\right)$ at $300 \mathrm{~K}^{6}{ }^{6}$ which is comparable to the resistivity of ITO thin films.

In condensed-matter physics, hyperfine experimental techniques $^{9,10}$ have been extensively employed to investigate structural, electronic, and magnetic properties at the atomic scale in pure and doped systems. ${ }^{11}$ In particular, the TimeDifferential Perturbed $\gamma-\gamma$ Angular-Correlation (TDPAC) spectroscopy provides a precise characterization of the electric-field-gradient (EFG) tensor at diluted (ppm) radioactive probe atoms, and therefore can be employed to elucidate the subnanoscopic environment of impurities or native atoms in solids. ${ }^{12-22}$ The EFG is a second-order symmetric tensor whose components are defined by $V_{i j}(\vec{r})=\left(\partial^{2} V(\vec{r})\right) /\left(\partial x_{i} \partial x_{j}\right)$, where $V(\vec{r})$ is the Coulomb potential generated by the charge density surrounding a probe nucleus. In the principal axis system, the EFG tensor is traceless. With the usual convention $\left|V_{33}\right| \geq\left|V_{22}\right| \geq\left|V_{11}\right|$, the EFG is completely defined by the largest component $V_{33}$ and the asymmetry parameter $\eta=\left(V_{11}\right.$ $\left.-V_{22}\right) / V_{33}$.

In a TDPAC experiment, a suitable probe atom is used, often an impurity in the system under study. At this probe site, the local information, in the case of electric-quadrupole interactions, is given by $\eta$ and the nuclear-quadrupole frequency $\omega_{Q}=e Q V_{33} / 4 I(2 I-1) \hbar . Q$ is the nuclear quadrupole moment of the sensitive nuclear state of the probe atom with spin $I$. In our case, $I=5 / 2$ for the ${ }^{181}$ Ta probe atom. The power of TDPAC lies in its highly local sensitivity to the anisotropic charge distribution close to the probe nucleus (due to the $r^{-3}$ dependence of the EFG from their charge sources), and therefore can give very precise information about the local environment of the probe atoms. All of the information that the EFG tensor can provide about the system under study, such as localization of impurities and defects, structural distortions, charge state of defect centers, etc., could be obtained by confrontation of the experimental results with theoretical predictions of the EFG. Very accurate EFG calculations in doped systems can nowadays be performed $^{16-18,20,23}$ through the use of all-electron (AE) ab initio

Received: May 16, 2014

Revised: July 24, 2014

Published: August 7, 2014 
electronic structure calculations in the framework of the Density Functional Theory (DFT). ${ }^{24,25}$

The crystallization process of amorphous $\mathrm{Sn}-\mathrm{O}$ thin films toward the desired most stable and transparent rutile $\mathrm{SnO}_{2}$ phase was studied using TDPAC, implanting ${ }^{181} \mathrm{Hf}\left(\rightarrow{ }^{181} \mathrm{Ta}\right)$ probe atoms, and conversion electron Mössbauer spectroscopy (CEMS) with ${ }^{119} \mathrm{Sn}$ as probes. ${ }^{26,27}$ From these studies, the authors concluded that the final stage of the process was crystalline rutile $\mathrm{SnO}_{2}$ and that Ta impurities did not affect the overall crystallization of the films. Despite the monochromaticity of the hyperfine interactions observed in the TDPAC experiments, the lack of an accurate EFG prediction at those times made it difficult to correctly predict the localization of the impurities in the host lattice, and if the impurities trap defects. In addition, the simple point-charge model (PCM) calculations used in ref 27 did not reproduce the observed $V_{33}$ and the $\eta$ parameter, and could not describe the local structural relaxations around the impurity and the origin of the temperature dependence of the EFG.

Recently, Qin et al. ${ }^{28}$ reported an ab initio study in Ta-doped rutile $\mathrm{SnO}_{2}$, focusing only on bulk (i.e., nonlocal) properties. However, due to the high Ta concentration used in that work, their results could not be compare with those coming from resistivity or TDPAC experiments. To our knowledge, a local characterization (experimental or theoretical) of the structural and electronic environment of Ta impurities in Ta-doped rutile $\mathrm{SnO}_{2}$ has not been reported yet. Hence, it is desirable to predict structural, electronic, and hyperfine properties of Ta-doped $\mathrm{SnO}_{2}$ with a concentration of dopants simulating isolated impurities, as is the case in TDPAC and the resistivity experiments. ${ }^{6}$

In this work, we perform a detailed first-principles study of structural, electronic, and hyperfine properties of Ta-doped rutile $\mathrm{SnO}_{2}$ with different doping concentrations using the state-of-the-art Full-Potential Augmented Plane Wave plus local orbitals (FP-APW+lo) method. ${ }^{29,30}$ Because the presence of intrinsic defects and/or codoping (with donor or acceptor impurities) can modify the impurity charge state, and as the electronic, structural, and hyperfine properties close to the Ta impurity may depend on this, ${ }^{18-20,23}$ we performed calculations for different charge states of the impurity. The predicted EFG tensors were compared to the EFGs obtained in the TDPAC experiments in ${ }^{181} \mathrm{Hf}\left(\rightarrow{ }^{181} \mathrm{Ta}\right)$-implanted $\mathrm{SnO}_{2}$ and $\mathrm{Sn}-\mathrm{O}$ thin films. ${ }^{26,27}$ Although the EFG can be a powerful tool to identify impurity charge states in doped systems, ${ }^{19,31-33}$ in the present study this identification is not trivial, and energetic studies were necessary to unravel this issue.

This Article is organized as follows. In section II, we describe the theoretical approach used. In section III.A, we present a detailed study of the undoped system and compare electronic and hyperfine properties with experimental results and other theoretical predictions from the literature. In sections III.B.1III.B.3, we present and discuss the structural relaxations, the electronic structure, and the EFG predictions, respectively, for different charge states of substitutional $\mathrm{Ta}$ impurities in rutile $\mathrm{SnO}_{2}$. In section III.B.4, we compare these ab initio results with experimental data and in section III.B.5 with other theoretical calculations. Finally, in section IV, we present our conclusions.

\section{THEORETICAL APPROACH AND CALCULATION DETAILS}

Rutile $\mathrm{SnO}_{2}$ is tetragonal with $a=b=4.7374(1) \AA$ and $c=$ $3.1864(1) \AA^{34,35}(c / a=0.672$ at room temperature and atmospheric pressure). The unit cell contains two molecules, with $\mathrm{Sn}$ atoms at positions $2 a,(0 ; 0 ; 0)$ and $(1 / 2 ; 1 / 2 ; 1 / 2)$, and the four $\mathrm{O}$ atoms at positions $4 f, \pm(u ; u ; 0)$ and $\pm(1 / 2+u ; 1 / 2-$ $u ; 1 / 2)$, with $u=0.3056(1)^{34}$ or $0.3064(4) .{ }^{35}$ Each $S n$ atom is surrounded by six nearest oxygen neighbors (ONN) forming an octahedron. The four oxygen atoms of the rectangular basal plane $(\mathrm{O} 1)$ are at a distance $d(\mathrm{Sn}-\mathrm{O} 1)=2.058 \AA$, and the two of the vertex are at $d(S n-02)=2.047 \AA$; see Figure 1a.

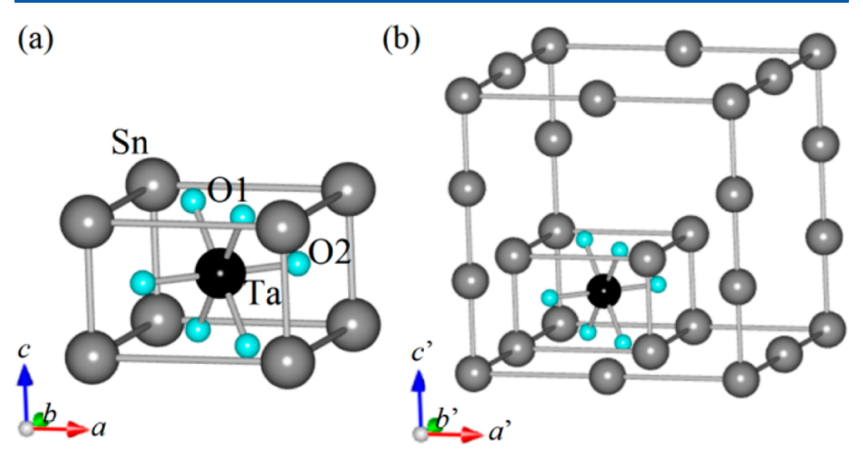

Figure 1. (a) Rutile $\mathrm{SnO}_{2}$ unit cell. (b) Supercell with $a^{\prime}=2 a, b^{\prime}=2 b$, $c^{\prime}=3 c$. The gray, black, and light-blue spheres correspond to $\mathrm{Sn}, \mathrm{Ta}$, and $\mathrm{O}$ atoms, respectively.

To simulate an isolated impurity, we constructed a supercell (SC) of 12 unit cells of $\mathrm{SnO}_{2}$ repeated periodically, in which one of the $24 \mathrm{Sn}$ atoms was replaced by a Ta atom, obtaining $\mathrm{Sn}_{0.958} \mathrm{Ta}_{0.042} \mathrm{O}_{2}$, representing a cationic doping of 4.2 at. \%. The resulting 72-atom SC is also tetragonal and has dimensions $a^{\prime}=$ $2 a=b^{\prime}=2 b=9.47 \AA$ and $c^{\prime}=3 c=9.56 \AA$ (see Figure $1 \mathrm{~b}$ ), with $c^{\prime} / a^{\prime}=1.01$, giving a distance of about $9.5 \AA$ between the $\mathrm{Ta}$ impurities. For checking purposes, we have also considered SCs containing $8\left(a^{\prime}=2 a=b^{\prime}=2 b=9.47 \AA\right.$ and $\left.c^{\prime}=2 c=6.37 \AA\right)$ and $16\left(a^{\prime}=2 a=b^{\prime}=2 b=9.47 \AA\right.$ and $\left.c^{\prime}=4 c=12.74 \AA\right)$ unit cells. It is worth mentioning that the presence of a Ta impurity at a $\mathrm{Sn}$ site breaks the equivalence between $\mathrm{O} 1$ and $\mathrm{O} 2$.

When a $\mathrm{Ta}^{5+}$ atom replaces a $\mathrm{Sn}^{4+}$ atom, Ta formally acts as a donor impurity. As we know, the charge state of the impurity could be essential in the description of the structural and electronic properties of the impurity-host system. Therefore, to describe real samples, where the presence of defects (vacancies, interstitial atoms, and/or the presence of substitutional donor or acceptor impurities), thermal effects, or optical transitions can change the charge state of the impurity, we performed calculations assuming two different physical situations:

(a) A Ta atom substitutes a $\mathrm{Sn}$ atom in the $\mathrm{SnO}_{2}$ supercell. We call this situation "neutral charge state", $\mathrm{SnO}_{2}: \mathrm{Ta}^{0}$.

(b) Taking into account the donor character of $\mathrm{Ta}$ when it replaces $\mathrm{Sn}$ in $\mathrm{SnO}_{2}$, we performed calculations removing one electron from the supercell. We will call this situation "charged state", $\mathrm{SnO}_{2}: \mathrm{Ta}^{+}$. For this physical situation, the presence of certain defects such as cation vacancies, interstitial oxygen atoms, acceptor impurities, or grain boundaries that could trap electrons is needed. Other sources of impurity ionization are thermal or optical transitions.

To solve the scalar-relativistic Kohn-Sham equations, in the framework of the DFT, ${ }^{24,25}$ we employed the FP-APW+lo method $^{29,30}$ implemented in the WIEN2k code. ${ }^{36}$ In this method, the wave functions are expanded in spherical harmonics inside non overlapping atomic spheres of radius $R_{\mathrm{MT}}$, and in plane waves in the interstitial region of the unit cell. 
Exchange and correlation effects were treated using the localdensity approximation (LDA), ${ }^{37}$ the generalized gradient approximation (GGA), ${ }^{38}$ and the GGA parametrization proposed by $\mathrm{Wu}$ and Cohen (WC-GGA). ${ }^{39}$ Because the differences between the results obtained with GGA and CWGGA are negligible, we only report the results using the LDA and CW-GGA approximations. The EFG tensor was obtained directly from the second derivative of the full potential. ${ }^{40,41}$ Structural optimizations were performed taking into account the quantum mechanically derived forces, and displacing all of the atoms in the SC according to a Newton damped scheme. ${ }^{42}$ The procedure was repeated until the forces on the ions were below $0.01 \mathrm{eV} / \AA ̊$. We used atomic spheres with $R_{\mathrm{MT}}(\mathrm{Ta})=$ $0.99 \AA, R_{\mathrm{MT}}(\mathrm{Sn})=0.95 \AA$, and $R_{\mathrm{MT}}(\mathrm{O})=0.86 \AA$. The parameter $R K_{\max }$ ( $R$ is the smallest muffin-tin radius and $K_{\max }$ is the largest wavenumber of the basis set), which controls the size of the basis set, was set to 7 . Integration in the reciprocal space was performed using the tetrahedron method considering $50 k$ points in the first Brillouin zone. A convergence less than $0.1 \times 10^{21} \mathrm{~V} / \mathrm{m}^{2}$ in the EFG components and $0.01 \AA$ in the interatomic distances was obtained using these parameters.

Finally, although the experimental TDPAC results ${ }^{26,27}$ do not suggest any magnetic interaction present at the $\mathrm{Ta}$ site in $\mathrm{SnO}_{2}:{ }^{181} \mathrm{Ta}$, we have also performed spin-polarized calculations for the doped system, and we checked that no magnetic solution exists at the equilibrium positions, for both charge states of the Ta impurity. Therefore, all of the results reported here correspond to non spin-polarized calculations.

\section{RESULTS AND DISCUSSION}

A. Undoped $\mathrm{SnO}_{2}$. Before studying the doped system, we studied first pure $\mathrm{SnO}_{2}$. To check the accuracy of the present calculations, we compared the predicted structural, hyperfine, and electronic properties with experimental data and theoretical results reported in the literature. For this purpose, we performed a complete optimization of the crystalline structure (see Table 1). From this optimization, we found for the internal

Table 1. Lattice $(a, c)$ and Internal Parameter $(u)$ Obtained Using FP-APW+lo in Both LDA and CW-GGA Calculations for Pure $\mathrm{SnO}_{2}$

\begin{tabular}{cccl} 
& $a(\AA)$ & $c(\AA)$ & \multicolumn{1}{c}{$u$} \\
experimental & $4.7374(1)^{a}$ & $3.1864^{a}$ & $0.3056(1)^{a}$ \\
& & & $0.3064(4)^{b}$ \\
LDA & 4.7297 & 3.1995 & 0.3061 \\
CW-GGA & 4.7710 & 3.1999 & 0.3056 \\
${ }^{a}$ Reference 34. ${ }^{b}$ Reference 35. & & \\
\hline
\end{tabular}

$u$ parameter a value of $u=0.3061$ for LDA and $u=0.3056$ for CW-GGA, differing by less than $1 \times 10^{-4}$ with respect to those obtained in X-ray diffraction (XRD) experiments, ${ }^{34,35}$ within errors (see Table 1). These values are in perfect agreement with those previously obtained ${ }^{43}$ using LAPW calculations $(u=$ 0.3060 for LDA and $u=0.3054$ for GGA). A rather higher value of $u=0.307$ was reported by $\mathrm{Qin}$ et al. ${ }^{28}$ using the CASTEP code and ultrasoft pseudopotentials. For the $a$ and $c$ lattice parameters, we found $a=4.7297 \AA$ and $c=3.1995 \AA$ for LDA, and $a=4.7710 \AA$ and $c=3.1999 \AA$ for CW-GGA (see Table 1).

In CEMS experiments, due to the nuclear spin $I=3 / 2$ of the sensitive state of the ${ }^{119} \mathrm{Sn}$ probe, a separate determination of $V_{33}$ and $\eta$ at the $\mathrm{Sn}$ site is not possible, the quadrupole splitting
$\Delta_{e Q}=\left(e Q V_{33}\left(1+\eta^{2} / 3\right)^{1 / 2}\right) / 2$ being the measured quantity. In Table 2 we show, adopting the experimental and optimized

Table 2. FP-APW+lo Predictions for the EFG Tensor Using the Experimental and Optimized Structures of Pure $\mathrm{SnO}_{2}$

$\begin{array}{clcccl} & & \begin{array}{c}V_{33} \\ \left(10^{21} \mathrm{~V} / \mathrm{m}^{2}\right)\end{array} & \eta & V_{33} \text { dir. } & \Delta_{e Q}{ }^{a}(\mathrm{~mm} / \mathrm{s}) \\ \text { exp. } & \text { LDA } & +5.06 & 0.49 & {[1 \overline{1} 0]} & -0.44 \\ \text { structure } & \text { CW-GGA } & +5.30 & 0.52 & {[1 \overline{1} 0]} & -0.46 \\ \text { opt. } & \text { LDA } & +5.71 & 0.44 & {[1 \overline{1} 0]} & -0.49 \\ \text { structure } & \text { CW-GGA } & +5.10 & 0.68 & {[1 \overline{1} 0]} & -0.46 \\ & \text { exp. } & & & & 0.499(13)^{c}\end{array}$

${ }^{a}$ To determine $\Delta e_{Q}$ from the predicted $V_{33}$, we used $Q\left({ }^{119} \mathrm{Sn}\right)=-132$ mb. ${ }^{44}{ }^{b}$ Reference $45 .{ }^{c}$ The sign of $\Delta_{e Q}$ has not been experimentally determined.

structures, the predictions of $V_{33}, \eta, V_{33}$ direction, and $\Delta_{e Q}$ at the $\mathrm{Sn}$ site, and compare with $\Delta_{e Q}$ coming from CEMS experiments (using the ${ }^{119} \mathrm{Sn}$ as probe). Using the most recent determination of the nuclear quadrupole moment of the excited state of ${ }^{119} \mathrm{Sn}, Q=-132 \mathrm{mb},{ }^{44}$ our predicted $\Delta_{e Q}$ values are in excellent agreement with the experimental value reported by Collins et al. ${ }^{45}$ for polycrystalline $\mathrm{SnO}_{2}$ (see Table 2). With this actualized $Q$ value, the $\Delta_{e Q}$ prediction by Errico ${ }^{43}$ using LAPW adopting the experimental lattice parameter and optimized $u$ value is also in perfect agreement with the experimental result.

Finally, in Table 3 we show the $p$ and d valence contributions to the EFG at the $\mathrm{Sn}$ site. The $\mathrm{s}-\mathrm{d}$ valence contributions are

Table 3. $p$ and $d$ Valence Contributions to the EFG Tensor Principal Components (in Units of $10^{21} \mathrm{~V} / \mathrm{m}^{2}$ ) at Sn Sites in Pure $\mathrm{SnO}_{2}$, Using the CW-GGA Approximation

\begin{tabular}{cccc} 
& $V_{11}$ & $V_{22}$ & $V_{33}$ \\
$\mathrm{p}$ & -0.44 & -3.65 & +4.09 \\
$\mathrm{~d}$ & -0.79 & -0.41 & +1.20 \\
\hline
\end{tabular}

negligible and are not shown here. The total density of states (DOS) and atom-projected partial density of states (PDOS), using CW-GGA, at $\mathrm{Sn}$ and $\mathrm{O}$ sites for pure $\mathrm{SnO}_{2}$ are shown in Figure 2. The fine structure of these results is independent of the approximation used for the exchange and correlation potential (LDA, GGA, and CW-GGA). The valence band (VB) has mainly O-2p character, showing hybridization with s-, p-, and $\mathrm{d}-\mathrm{Sn}$ orbitals, in agreement with previous DFT results, ${ }^{28,43,46}$ and the expected insulator behavior was obtained. At lower energies (from -19 to $-15 \mathrm{eV}$ ) the hybridization of $\mathrm{Sn}-4 \mathrm{~d}$ with O-2s states is observed, in excellent agreement with $\mathrm{X}$-ray photoelectron spectroscopy (XPS) measurements ${ }^{47}$ and the results reported by Dixit et al. ${ }^{46}$ Finally, the conduction band $(\mathrm{CB}$ ) is described by $\mathrm{Sn}-5 \mathrm{~s}$ and $\mathrm{O}-2 \mathrm{p}$ (with a little contribution of $\mathrm{O}-2 \mathrm{~s}$ ).

Our theoretical band gap is $2.5 \mathrm{eV}$, in good agreement with other DFT results, ${ }^{43,48}$ but smaller than the experimental value of $3.6 \mathrm{eV} .^{47,49,50}$ The underestimation of the band gap is a wellknown deficiency in DFT calculations, but this does not interfere with the calculations of ground-state properties like the EFG. Nevertheless, our value is notably larger than the almost negligibly band gap reported in ref 28 for pure $\mathrm{SnO}_{2}$. This underestimation of the band gap can be resolved using the Tran-Blaha modified Becke Johnson (TB-mBJ) potential, giving a value of $3.5 \mathrm{eV}$, in excellent agreement with the experimental gap and with that predicted by Dixit et al. ${ }^{46}$ The 


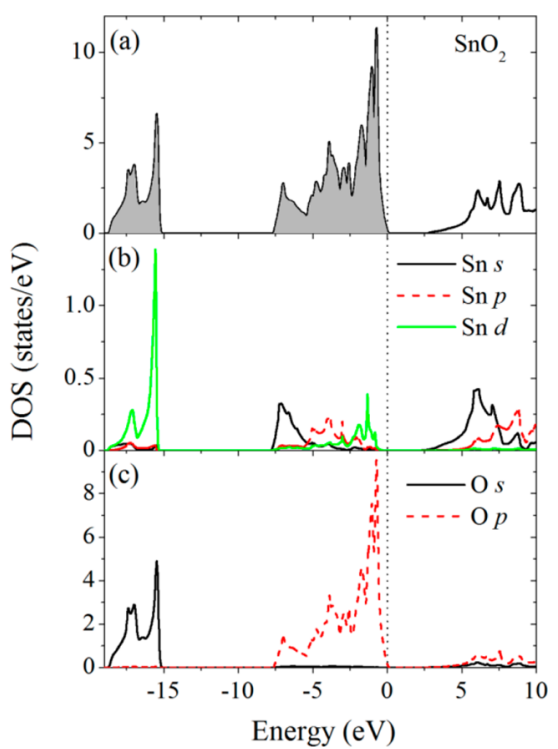

Figure 2. (a) Total density of states (DOS) for pure $\mathrm{SnO}_{2}$. The gray area shows the occupied states. Atom-resolved partial DOS of (b) s, p, and $d$ contributions projected at $S n$ site and (c) $s$ and p contributions projected at $\mathrm{O}$ sites for pure $\mathrm{SnO}_{2}$. All cases correspond to CW-GGA. The energy is referred to the last occupied state (vertical dotted line).

EFG can be obtained, using this approximation, in pure oxides, ${ }^{51}$ but the forces acting on the atoms cannot be calculated. Hence, due to this restriction, it is not possible to optimize the structure nor to predict the equilibrium atomic positions in doped systems, and therefore accurately predict the EFG at impurity sites using the TB-mBJ potential.

B. Ta-Doped $\mathrm{SnO}_{2}$. 1. Structural Relaxations. The substitution of an indigenous $\mathrm{Sn}$ atom by a $\mathrm{Ta}$ impurity produces not negligible forces on its nearest neighbor atoms. As a first step, we performed a complete structural optimization of the lattice and internal positions in the $2 \times 2 \times 1,2 \times 2 \times 2$, and $2 \times 2 \times 3 \mathrm{SnO}_{2}: \mathrm{Ta}^{0} \mathrm{SCs}$. We found the absence of volume variation in the $2 \times 2 \times 3$ SC. A little expansion appears when Ta concentration increases, being $1 \%$ at the $2 \times 2 \times 1$ SC. On the other hand, we checked that the final equilibrium structures, and therefore the electronic structure, are independent of the initial positions (i.e., using the experimental or optimized structure of pure $\mathrm{SnO}_{2}$ as starting point). This is due to the strong displacement of the impurity's nearest neighbors relative to the subtle position refinement produced in the structural optimization process.

In Table 4 we compare the $\mathrm{Ta}-\mathrm{O} 1$ and $\mathrm{Ta}-\mathrm{O} 2$ bondlengths at the final equilibrium positions for $\mathrm{SnO}_{2}: \mathrm{Ta}^{0}$ and $\mathrm{SnO}_{2}: \mathrm{Ta}^{+}$with the experimental $\mathrm{Sn}-\mathrm{O} 1$ and $\mathrm{Sn}-\mathrm{O} 2$ bond- lengths for pure $\mathrm{SnO}_{2}$. We see that for both charge states of the impurity the ONN relaxes inward, shortening the Ta-ONN bond-lengths. The magnitude of these relaxations is nearly independent of the charge state of the impurity (as occurs in Ta-doped rutile $\left.\mathrm{TiO}_{2}\right)^{20}$ These contractions are quite anisotropic, with the equilibrium $\mathrm{Ta}-\mathrm{O} 2$ distance larger than the $\mathrm{Ta}-\mathrm{O} 1$ distance, opposite to the initial unrelaxed structure. This anisotropy can be understood inspecting the relaxations of $\mathrm{O} 2$ and $\mathrm{O} 1$ in the $(1 \overline{1} 0)$ and (110) planes, respectively. In the (110) plane, a shortening of the $\mathrm{Ta}-\mathrm{O} 2$ bonds implies a considerable stretching in the $\mathrm{Sn}-\mathrm{O} 2$ bonds (see Figure 3a). (a)

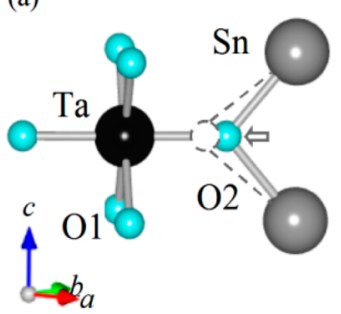

(b)

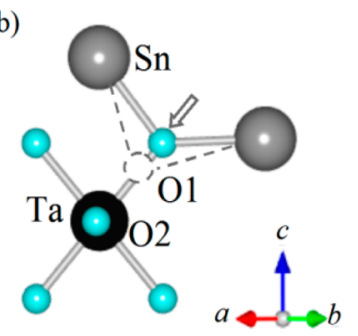

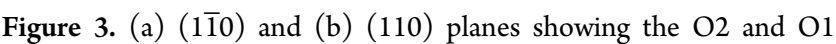
coordination, respectively. The arrow indicates the $\mathrm{O} 2$ and $\mathrm{O} 1$ displacement. The dashed lines indicate the final equilibrium positions for $\mathrm{SnO}_{2}: \mathrm{Ta}^{0}$. The contractions were magnified to better visualize the effect.

However, in the (110) plane, a shortening of the Ta-O1 bonds produces a weaker stretching of the $\mathrm{Sn}-\mathrm{O} 1$ bonds (Figure $3 \mathrm{~b}$ ) due to the geometry involved in the cationic coordination at $\mathrm{O} 1$ and $\mathrm{O} 2$ atomic sites. In the same way, because the structure is more "open" in the $\mathrm{Ta}-\mathrm{O} 1$ direction than in the $\mathrm{Ta}-\mathrm{O} 2$ axis (i.e., the angle $\mathrm{Sn}-\mathrm{O} 1-\mathrm{Sn}$ is larger than the angle $\mathrm{Sn}-\mathrm{O} 2-\mathrm{Sn}$; see Figure $3 \mathrm{~b}$ ), the forces on $\mathrm{O} 1$ produced by the two nearest $\mathrm{Sn}$ neighbor atoms along the $\mathrm{Ta}-\mathrm{O} 1$ direction are weaker than those acting on $\mathrm{O} 2$ along the $\mathrm{Ta}-\mathrm{O} 2$ direction, allowing a stronger shortening of the $\mathrm{Ta}-\mathrm{O} 1$ bond-length. These contractions follow the general trend already observed in other Ta-doped binary oxides; ${ }^{20,31}$ that is, the impurity tries to reconstruct the ONN bond-length that it has in its most stable oxide. In the present case, the $\mathrm{Ta}-\mathrm{ONN}$ bond-lengths in $\mathrm{TaO}_{2}$ (rutile) and $\mathrm{Ta}_{2} \mathrm{O}_{5}$ (the most stable tantalum oxide) are approximately 2.02 and $2.00 \AA$, respectively, very close to final $\mathrm{Ta}-\mathrm{ONN}$ distances at the final equilibrium positions (see Table 4).

The contractions in the bond-lengths produced by $\mathrm{Ta}$ at the Sn site are related to the fact that the ionic radius for 6-fold coordinated $\mathrm{Ta}^{5+}(0.65 \AA)$ is smaller than the ionic radius for 6fold coordinated $\mathrm{Sn}^{4+}(0.69 \AA) .{ }^{52}$ In rutile $\mathrm{TiO}_{2}$, both $\mathrm{Ta}$ and

Table 4. Experimental $\mathrm{Sn}-\mathrm{O} 1$ and $\mathrm{Sn}-\mathrm{O} 2$ Bond-Lengths for Pure $\mathrm{SnO}_{2}$ and Predicted Equilibrium $\mathrm{Ta}-\mathrm{O} 1$ and $\mathrm{Ta}-\mathrm{O} 2$ Distances for $\mathrm{SnO}_{2}: \mathrm{Ta}^{0}$ and $\mathrm{SnO}_{2}: \mathrm{Ta}^{+}$

\begin{tabular}{|c|c|c|c|c|}
\hline & \multicolumn{4}{|c|}{$\mathrm{SnO}_{2}$} \\
\hline & $d(\mathrm{Sn}-\mathrm{O} 1)$ & & & $d(\mathrm{Sn}-\mathrm{O} 2)(\AA)$ \\
\hline \multirow[t]{4}{*}{ exp. } & 2.058 & & & 2.047 \\
\hline & \multicolumn{4}{|c|}{$\mathrm{SnO}_{2}: \mathrm{Ta}$} \\
\hline & \multicolumn{2}{|c|}{$\mathrm{SnO}_{2}: \mathrm{Ta}^{0}$} & \multicolumn{2}{|c|}{$\mathrm{SnO}_{2}: \mathrm{Ta}^{+}$} \\
\hline & $d(\mathrm{Ta}-\mathrm{O} 1)(\AA)$ & $d(\mathrm{Ta}-\mathrm{O} 2)(\AA)$ & $d(\mathrm{Ta}-\mathrm{O} 1)(\AA)$ & $d(\mathrm{Ta}-\mathrm{O} 2)(\AA)$ \\
\hline LDA & 1.982 & 1.995 & 1.982 & 1.996 \\
\hline WC-GGA & 1.985 & 1.995 & 1.987 & 1.999 \\
\hline
\end{tabular}


$\mathrm{Cd}$ impurities have ionic radii larger than that of $\mathrm{Ti}$ producing relaxations that move the ONN outward, enlarging the impurity-ONN bond-length. ${ }^{19,20}$ To illustrate this point, we plot in Figure 4 the ratio $\Delta d / d_{0}\left(\Delta d=d-d_{0}, d\right.$ is the

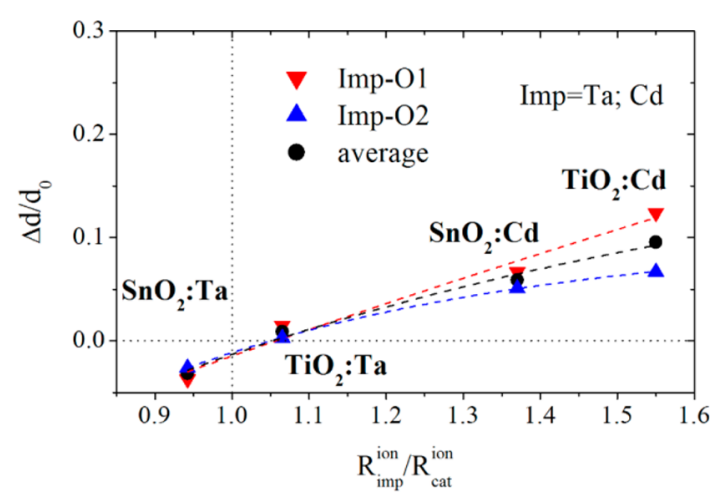

Figure 4. Relative bond-length relaxation of the nearest oxygen atoms of the impurities, $\Delta d / d_{0}$, as a function of the ratio $R_{\mathrm{imp}}^{\text {ionic }} / R_{\mathrm{cat}}^{\text {ionic }}$ in rutile $\mathrm{SnO}_{2}$ and $\mathrm{TiO}_{2} . d_{0}$ is the cation-ONN bond-length of the undoped system and $\Delta d=d-d_{0}$, where $d$ is the impurity-ONN bond-length in the relaxed structure. $R_{\mathrm{imp}}^{\text {ionic }}$ and $R_{\mathrm{cat}}^{\text {ionic }}$ are the ionic radii of the impurity and the host cation, respectively. The equilibrium structures of the doped systems correspond to the charge state of the SC that reproduces the experimental EFG tensor in each case.

impurity-ONN bond-length in the doped system and $d_{0}$ is the cation-ONN bond-length in the pure system) as a function of $R_{\text {imp }}^{\text {ionic }} / R_{\text {cat }}^{\text {ionic }}\left(R_{\text {imp }}^{\text {ionic }}\right.$ and $R_{\text {cat }}^{\text {ionic }}$ are the ionic radii of the impurity and the host cation, respectively) for $\mathrm{Ta}$ and $\mathrm{Cd}$ impurities in rutile $\mathrm{SnO}_{2}$ and $\mathrm{TiO}_{2}$. This plot shows a positive proportionality between the magnitude of the structural relaxations (relative to the unrelaxed system) and the ionic radii of the impurity (relative to the native cation). This relationship was already observed in oxides with the corundum structure $(\alpha$ $\mathrm{Al}_{2} \mathrm{O}_{3}, \alpha-\mathrm{Fe}_{2} \mathrm{O}_{3}$, and $\mathrm{Cr}_{2} \mathrm{O}_{3}$ ) for both Ta and $\mathrm{Cd}$ impurities. ${ }^{53}$ In the last case, due to the wider range of $R_{\text {cat }}^{\text {ionic }}$ studied, it is possible to see an asymptotic behavior of the bond-length relaxations for $R_{\mathrm{imp}}^{\text {ionic }} / R_{\mathrm{cat}}^{\text {ionic }}>1.55$.

2. Electronic Structure. In addition to the structural relaxations, the substitution of a $\mathrm{Sn}$ atom by a Ta impurity (at pure $\mathrm{SnO}_{2}$ positions) induces strong changes in the electronic structure of the semiconducting host. The Ta atom introduces a donor impurity level located at the bottom of the $\mathrm{CB}$, as can be seen comparing the DOS of Figures $2 \mathrm{a}$ and 5, turning metallic the system $\mathrm{SnO}_{2}: \mathrm{Ta}^{0}$. In Figure $5 \mathrm{~b}$, we can see that the Ta-d states contribute to both the VB and the impurity level in the CB. A minor Ta-p contribution appears only in the VB. Integration of the total DOS of the occupied impurity states in the $\mathrm{CB}$ gives a value of $0.98 \mathrm{e}^{-}$, which agrees with the nominal donor character of $\mathrm{Ta}^{5+}$ substituting a $\mathrm{Sn}^{4+}$ atom in $\mathrm{SnO}_{2}$. Figure 6a shows the total DOS and the atom-resolved PDOS projected at $\mathrm{Ta}, \mathrm{Sn}$, and $\mathrm{O}$ sites in the impurity level energy region in $\mathrm{SnO}_{2}: \mathrm{Ta}^{0}$. This impurity level is composed of Ta-5d, Sn-5s, and O-2p orbital symmetries, with $0.22 \mathrm{e}^{-}$(of the $0.98 \mathrm{e}^{-}$that have the impurity level until the last occupied state) corresponding to the $\mathrm{Ta}$ states. When an electron is removed from the SC, the impurity level becomes empty and the resulting system $\left(\mathrm{SnO}_{2}: \mathrm{Ta}^{+}\right)$insulating.

After the structural relaxations, the electronic structure at the bottom of the $\mathrm{CB}$ in $\mathrm{SnO}_{2}: \mathrm{Ta}^{0}$ changes: the Ta-d impurity level is shifted to higher energies (see Figure 6) and only $0.02 \mathrm{e}^{-}$

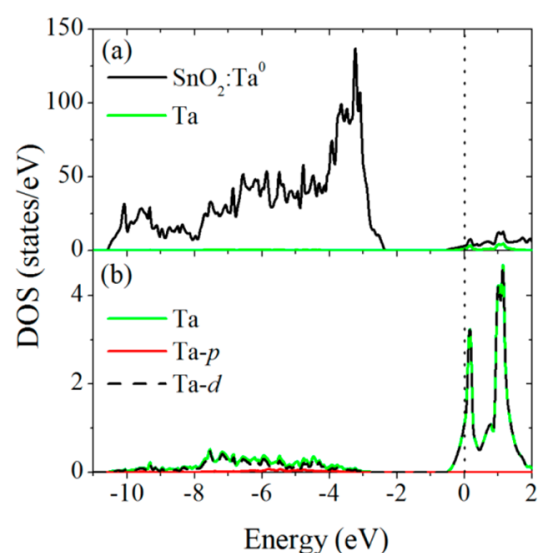

Figure 5. (a) Total density of states (DOS) and atom-resolved partial DOS (PDOS) projected at Ta site and (b) PDOS of $\mathrm{p}$ and $\mathrm{d}$ contributions projected at Ta site, in unrelaxed $\mathrm{SnO}_{2}: \mathrm{Ta}^{0}$. The energy is referred to the last occupied state (vertical dotted line).

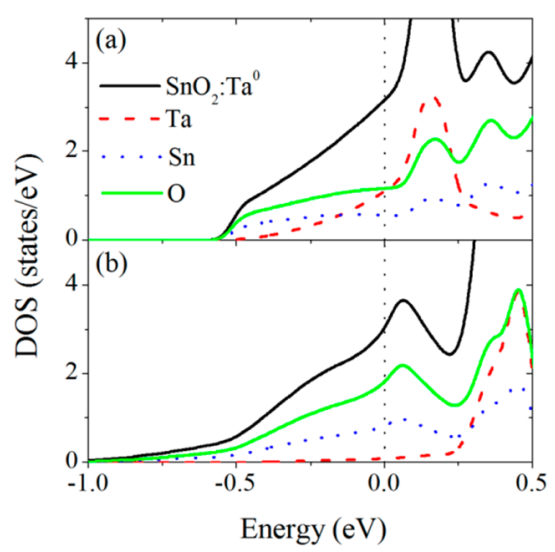

Figure 6. Total density of states (DOS) and atom-resolved partial DOS (PDOS) projected at $\mathrm{Ta}, \mathrm{Sn}$, and $\mathrm{O}$ sites in the impurity energy region in (a) unrelaxed $\mathrm{SnO}_{2}: \mathrm{Ta}^{0}$ and (b) relaxed $\mathrm{SnO}_{2}: \mathrm{Ta}^{0}$. The energy is referred to the last occupied state (vertical dotted line).

remain at the Ta sphere. The same effect was observed in $\mathrm{TiO}_{2}: \mathrm{Ta}^{20}$ In effect, in this system $0.03 \mathrm{e}^{-}$remain in the Ta-d states located at the impurity level after structural relaxation. In Figure 7 we show the d-PDOS projected at Ta site for $\mathrm{SnO}_{2}: \mathrm{Ta}^{0}$ and $\mathrm{SnO}_{2}: \mathrm{Ta}^{+}$systems, before and after the structural relaxations in the impurity level energy region. Before relaxations, only $\mathrm{d}_{z}{ }^{2}$ and $\mathrm{d}_{x^{2}-y^{2}}$ Ta symmetries are occupied in
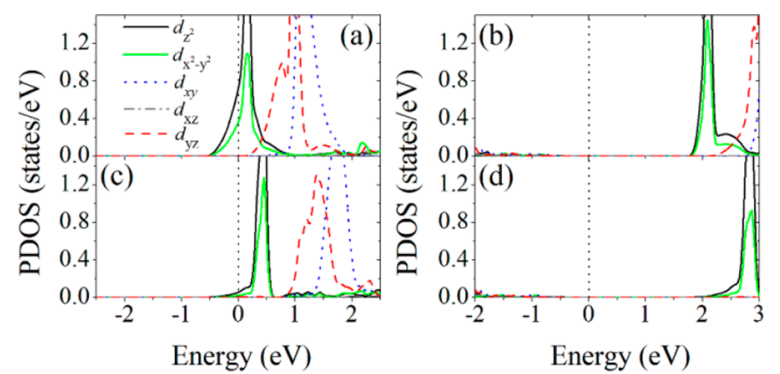

Figure 7. Partial density of states (PDOS) of $\mathrm{d}$ contributions projected at $\mathrm{Ta}$ site in the impurity level energy region for (a) unrelaxed $\mathrm{SnO}_{2}: \mathrm{Ta}^{0}$, (b) unrelaxed $\mathrm{SnO}_{2}: \mathrm{Ta}^{+}$, (c) relaxed $\mathrm{SnO}_{2}: \mathrm{Ta}^{0}$, and (d) relaxed $\mathrm{SnO}_{2}: \mathrm{Ta}^{+}$. The energy is referred to the last occupied state (vertical dotted line). 
the case of $\mathrm{SnO}_{2}: \mathrm{Ta}^{0}$ (see Figure 7a), and became empty when one electron is removed from the SC (see Figure $7 \mathrm{~b}$ ). After relaxation in $\mathrm{SnO}_{2}: \mathrm{Ta}^{0}$, the $\mathrm{d}_{z}{ }^{2}$ and $\mathrm{d}_{x^{2}-y^{2}}$ symmetries shift to higher energies (Figure 7c), and are practically empty even before the removal of the $\mathrm{e}^{-}$from the SC (see Figure 7d).

In Figure $8 \mathrm{a}$ and $\mathrm{b}$ we show the electron density $\rho^{\mathrm{val}}(\mathbf{r})$ at the (110) and (110) planes, respectively, for pure $\mathrm{SnO}_{2}$, and in
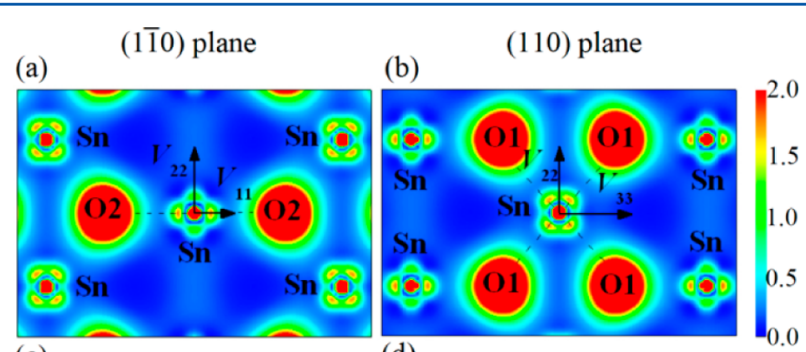

(c)

(d)

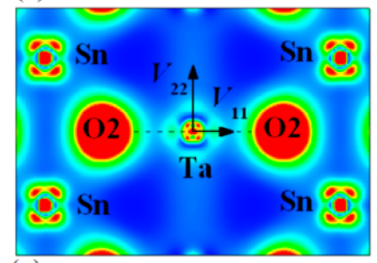

(e)

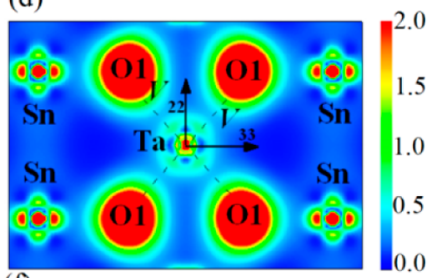

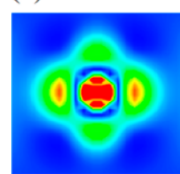

Sn site

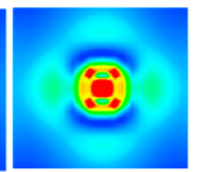

Ta site

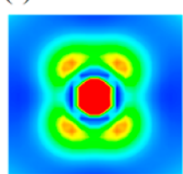

Sn site

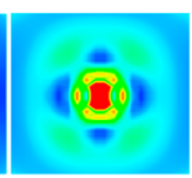

Ta site
Figure 8. Valence electron densities $\rho^{\mathrm{val}}(\mathbf{r})$ for $(\mathrm{a}, \mathrm{b})$ undoped $\mathrm{SnO}_{2}$ and $(\mathrm{c}, \mathrm{d})$ unrelaxed $\mathrm{SnO}_{2}: \mathrm{Ta}^{0}$. Parts (a) and (c) correspond to the $(1 \overline{1} 0)$ plane, whereas $(\mathrm{b})$ and $(\mathrm{d})$ refer to the $(110)$ plane. We show the EFG orientation in each plane. Comparison between magnified $\rho^{\text {val }}(\mathbf{r})$ at $\mathrm{Sn}$ site for undoped $\mathrm{SnO}_{2}$ and at $\mathrm{Ta}$ impurity site for unrelaxed $\mathrm{SnO}_{2}: \mathrm{Ta}^{0}$ in the (e) (11̄0) and (f) (110) planes.

Figure $8 \mathrm{c}$ and $\mathrm{d}$ for unrelaxed $\mathrm{SnO}_{2}: \mathrm{Ta}^{0}$ at both planes, respectively. To understand the effect produced by the Ta impurity in the electronic charge distribution, we compare in Figure $8 \mathrm{e}$ and $\mathrm{f} \rho^{\mathrm{val}}(\mathbf{r})$ at $\mathrm{Sn}$ and $\mathrm{Ta}$ sites. From these comparisons, we can see a transition from a bonding- to an antibonding-like charge distribution at the cationic site, when Ta replaces $\mathrm{Sn}\left(\mathrm{SnO}_{2}: \mathrm{Ta}^{0}\right)$.

Figure $9 \mathrm{a}$ and $\mathrm{b}$ shows the electron density $\rho^{\text {imp }}(\mathbf{r})$ for the energy region of the impurity level (from -0.6 to $0 \mathrm{eV}$; see Figure 5a) in unrelaxed $\mathrm{SnO}_{2}: \mathrm{Ta}^{0}$, for both $(1 \overline{1} 0)$ and (110) planes, respectively. The $0.22 \mathrm{e}^{-}$with $\mathrm{Ta}$ d-character at the partially filled impurity level have a pure antibonding-like distribution and are localized at the impurity atom, giving the main antibonding contribution to the total electron density described before in Figure $8 \mathrm{c}$ and $\mathrm{d}$. The bonding contribution to $\rho(\mathbf{r})$ at the Ta site comes from the hybridization of $\mathrm{Ta}$ and $\mathrm{O}$ states of the VB (from -10.6 to $-2.4 \mathrm{eV}$; see Figure $5 \mathrm{~b}$ ). In Figure $9 \mathrm{a}$ and $\mathrm{b}$, we can see that the rest of the electrons belonging to the impurity level $\left(0.76 \mathrm{e}^{-}\right)$are localized at the $\mathrm{Sn}$ and $\mathrm{O}$ atoms of the SC, as was already seen in Figure 6a through the inspection at the atom-resolved PDOS at the impurity level. To view the effect produced by the relaxation, we compare $\rho^{\text {imp }}(\mathbf{r})$ at Ta sites for unrelaxed and relaxed $\mathrm{SnO}_{2}: \mathrm{Ta}^{0}$ in the $(1 \overline{1} 0)$ (Figure 9c) and (110) (Figure 9d) planes. From these comparisons, we can see that after the structural relaxation, almost all of the electronic charge at Ta (a)

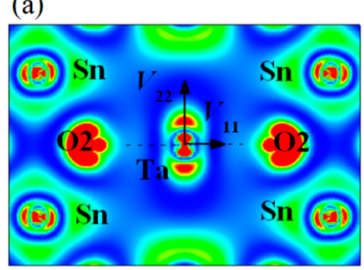

(c)

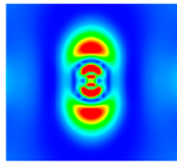

unrelaxed

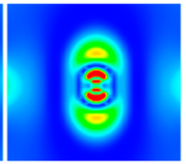

relaxed

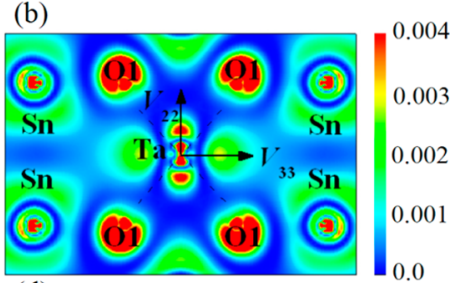

(d)

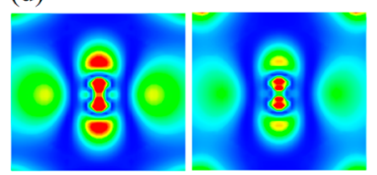

unrelaxed relaxed
Figure 9. Electron densities $\rho^{\mathrm{imp}}(\mathbf{r})$ in the energy range of the impurity level for unrelaxed $\mathrm{SnO}_{2:} \mathrm{Ta}^{0}$ in the (a) (11̄0) and (b) (110) planes. We show the EFG orientation in each plane. Comparison between magnified $\rho^{\mathrm{imp}}(\mathbf{r})$ at Ta site for unrelaxed and relaxed $\mathrm{SnO}_{2:} \mathrm{Ta}^{0}$ in the (c) (110) and (d) (110) planes.

disappears. This charge must move to the oxygen atoms, while that from the Sn atoms remains unaltered, as can be deduced from the shift of the Ta states to higher energies, the increase of the oxygen contribution, and the constancy of the $\mathrm{Sn}$ states at the impurity level after the structural relaxation (see Figure 6).

3. EFG. Let us now discuss the FP-APW+lo results for the EFG tensor. As can be seen comparing Tables 2 and 5, the substitution of a $\mathrm{Sn}$ atom by a Ta impurity in the $\mathrm{SnO}_{2}: \mathrm{Ta}^{0}$ neutral cell (at the positions of the undoped system) produces significant changes in the EFG tensor. $V_{33}$ increases approximately from 5 to $15 \times 10^{21} \mathrm{~V} / \mathrm{m}^{2}$, the asymmetry parameter $\eta$ increases up to 0.9 , whereas the direction of $V_{33}$ does not change. These changes are correlated with the different electronic structure of the impurity as compared to that of the Sn atom. When we remove one electron from the $\mathrm{SnO}_{2}: \mathrm{Ta}^{0} \mathrm{SC}$ (at fixed positions), the changes in the EFG are purely due to electronic effects. In effect, $V_{33}$ grows to $21 \times 10^{21}$ $\mathrm{V} / \mathrm{m}^{2}$ and $\eta$ decreases to 0.2 , as Table 5 shows.

Table 6 presents the EFG predictions at the equilibrium structures of $\mathrm{SnO}_{2}: \mathrm{Ta}^{0}$ and $\mathrm{SnO}_{2}: \mathrm{Ta}^{+}$, that is, after the structural relaxation. First, we can see here a small difference in the EFG parameters obtained with CW-GGA and LDA for each charge state of the impurity. The differences between LDA and CW-GGA can be associated with the small differences in the final equilibrium structures predicted by each approximation. Indeed, if we performed WC-GGA calculations using the equilibrium positions obtained with $\mathrm{LDA}$ in $\mathrm{SnO}_{2}: \mathrm{Ta}^{0}$ and $\mathrm{SnO}_{2}: \mathrm{Ta}^{+}$, we obtain $V_{33}=+16.01 \times 10^{21} \mathrm{~V} / \mathrm{m}^{2}$ and $\eta=0.95$, and $V_{33}=+17.55 \times 10^{21} \mathrm{~V} / \mathrm{m}^{2}$ and $\eta=0.90$, respectively. These values are very similar to the results obtained with the LDA approximation (see Table 6, first row). These results indicate that for the same structural positions both exchange and correlation potentials predict the same EFG tensor, pointing to the same description of the electronic structure for Ta-doped $\mathrm{SnO}_{2}$ by both approximations.

Opposite to the case of the unrelaxed system (see Table 5), it is interesting to notice that the resulting EFG values at the equilibrium positions are very similar for both charge states of the impurity (see Table 6). Again, the small differences in the EFG values predicted by LDA and CW-GGA are due to the small differences in the final equilibrium structures predicted by each approximation. On the other hand, the small differences in the EFG values for a given approximation (LDA or CW-GGA) 
Table 5. FP-APW+lo Predictions for $V_{33}$ and the Asymmetry Parameter $\boldsymbol{\eta}$ at Ta Sites in Unrelaxed Ta-Doped SnO $\mathrm{T}_{2}$ for the Two Charge States Studied

\begin{tabular}{|c|c|c|c|c|c|c|}
\hline & \multicolumn{3}{|c|}{$\mathrm{SnO}_{2}: \mathrm{Ta}^{0}$} & \multicolumn{3}{|c|}{$\mathrm{SnO}_{2}: \mathrm{Ta}^{+}$} \\
\hline & $V_{33}\left(10^{21} \mathrm{~V} / \mathrm{m}^{2}\right)$ & $\eta$ & $V_{33}$ dir. & $V_{33}\left(10^{21} \mathrm{~V} / \mathrm{m}^{2}\right)$ & $\eta$ & $V_{33}$ dir. \\
\hline LDA & +15.64 & 0.89 & {$[1 \overline{1} 0]$} & +21.09 & 0.23 & {$[1 \overline{1} 0]$} \\
\hline WC-GGA & +15.99 & 0.89 & {$[1 \overline{1} 0]$} & +21.37 & 0.26 & {$[1 \overline{1} 0]$} \\
\hline
\end{tabular}

Table 6. FP-APW+lo Predictions of $V_{33}$ and the Asymmetry Parameter $\eta$ at Ta Sites in Relaxed Ta-Doped SnO $\mathrm{T}_{2}$ for the Two Charge States Studied

\begin{tabular}{|c|c|c|c|c|c|c|}
\hline & \multicolumn{3}{|c|}{$\mathrm{SnO}_{2}: \mathrm{Ta}^{0}$} & \multicolumn{3}{|c|}{$\mathrm{SnO}_{2}: \mathrm{Ta}^{+}$} \\
\hline & $V_{33}\left(10^{21} \mathrm{~V} / \mathrm{m}^{2}\right)$ & $\eta$ & $V_{33}$ dir. & $V_{33}\left(10^{21} \mathrm{~V} / \mathrm{m}^{2}\right)$ & $\eta$ & $V_{33}$ dir. \\
\hline LDA & +16.65 & 0.95 & {$[1 \overline{1} 0]$} & +17.67 & 0.89 & {$[1 \overline{1} 0]$} \\
\hline WC-GGA & +17.06 & 0.80 & {$[1 \overline{1} 0]$} & +17.25 & 0.83 & {$[1 \overline{1} 0]$} \\
\hline $\exp ^{a}$ & $16.96(2)$ & 0.7 & \multicolumn{2}{|c|}{ measured at room temp } & & \\
\hline & $16.91(2)$ & 0.7 & \multicolumn{2}{|c|}{ measured at $1173 \mathrm{~K}$} & & \\
\hline $\exp ^{b}$ & $17.02(3)$ & $0.72(1)$ & \multicolumn{2}{|c|}{ measured at room temp } & & \\
\hline
\end{tabular}

${ }^{a}$ Reference 27. ${ }^{b}$ Reference 26.

between $\mathrm{SnO}_{2}: \mathrm{Ta}^{0}$ and $\mathrm{SnO}_{2}: \mathrm{Ta}^{+}$in the relaxed systems are mainly due to electronic effects. To probe this, we took the equilibrium structure of $\mathrm{SnO}_{2}: \mathrm{Ta}^{0}$ obtained with the LDA approximation, and performed calculations (also with LDA) removing one electron from the $\mathrm{SC}$ at this fixed atomic position. We thus obtained $V_{33}=+17.82 \times 10^{21} \mathrm{~V} / \mathrm{m}^{2}$ and $\eta=$ 0.86 , which is the value (within the convergence error) obtained for the relaxed structure of $\mathrm{SnO}_{2}: \mathrm{Ta}^{+}$(see Table 6).

Comparing Tables 5 and 6 , we can see that the relaxation process, for the neutral charge state, produces small changes in the EFG (see first columns of both tables), while in $\mathrm{SnO}_{2}: \mathrm{Ta}^{+}$ the change is drastic: $V_{33}$ reduces from 21 to $17 \times 10^{21} \mathrm{~V} / \mathrm{m}^{2}$ and $\eta$ increases from 0.2 to 0.8 (see last columns of Tables 5 and 6). To understand this intriguing behavior, we will analyze in detail the $\mathrm{p}$ and $\mathrm{d}$ valence contribution to the EFG, the electron charge density around the Ta impurity in each system, and the PDOS projected at the impurity site, where the EFG has its origin. Table 7 shows the $\mathrm{p}$ and $\mathrm{d}$ valence contributions

Table 7. $p$ and $d$ Valence Contributions to the EFG Tensor Principal Components (in Units of $10^{21} \mathrm{~V} / \mathrm{m}^{2}$ ) at Ta Sites in Ta-Doped $\mathrm{SnO}_{2}$ for Unrelaxed and Relaxed Structures of the Neutral and Charged Supercells

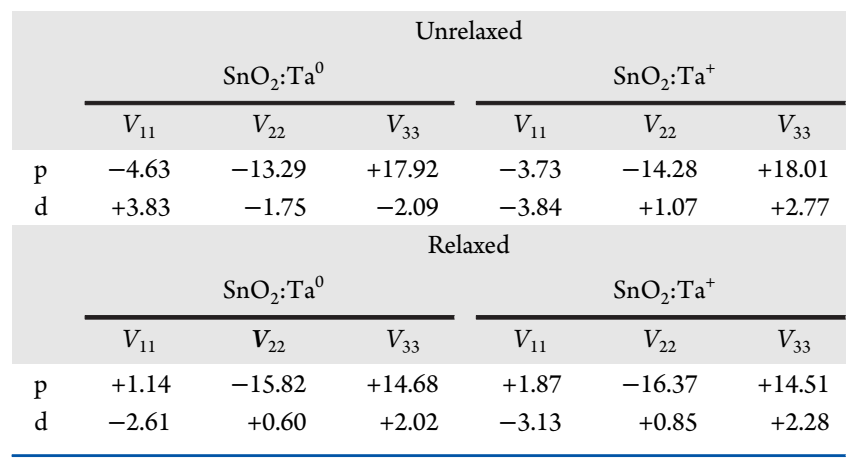

to the EFG at the Ta site in $\mathrm{SnO}_{2}: \mathrm{Ta}^{0}$ and $\mathrm{SnO}_{2}: \mathrm{Ta}^{+}$for both unrelaxed and relaxed structures. The $s-d$ and $f$ valence contributions are negligible and are not shown here. As we can see, the Ta-p contribution dominates. Nevertheless, the $d$ contribution plays an important role.

When the $\mathrm{SnO}_{2}: \mathrm{Ta}^{0}$ system is relaxed, the decrease in the $\mathrm{p}$ contribution is compensated by an increase of the $d$ - contribution, and therefore $V_{33}$ practically does not change. This change in the d-contribution is mainly due to the fact that the Ta-d symmetries at the impurity level became unoccupied (they shift to higher energies above the last occupied state; see Figure $7 a$ and $c$ ), and the relative weight of different $d-$ symmetries along the VB energy region does not change. On the other hand, the change in the p-contribution is originated in changes in the relative weight of different p-symmetries along the VB energy region. It is interesting to notice that, in general, changes in the EFG at the impurity site cannot be understood by inspecting only the impurity level region (shown in Figure 7 ), because the EFG results from the integration of the atomprojected PDOS, basically $\mathrm{p}$ - and d-states, at the impurity site over the entire energy range (valence band and impurity level). ${ }^{23}$

When we remove one electron from the unrelaxed SC (see Figure $7 a$ and $b$ ), there is no change in the relative weight of different $\mathrm{p}$ and $\mathrm{d}$ symmetries along the valence energy region, but the $\mathrm{d}_{z^{2}}$ - and $\mathrm{d}_{x^{2}-y^{2}}$-Ta symmetries in the impurity level that have 0.22 electrons become empty, changing greatly the $d$ contribution to the EFG.

On the other hand, the great changes produced when $\mathrm{SnO}_{2}: \mathrm{Ta}^{+}$relaxes are mainly due to changes in the relative weights of different Ta $\mathrm{p}$ symmetries along the VB energy region.

Finally, when one electron is removed from the SC in the relaxed system, the small change in the EFG (see Table 6) is due to small changes in the d-contributions to the EFG (see Table 7). In this case, the change in the $\mathrm{d}$ contribution is due to the removal of a small amount of charge ( 0.02 electrons) with particular Ta d-symmetries in the impurity level (see Figure 7c and $\mathrm{d}$ ).

4. Comparison with Experimental Results (Resistivity and TDPAC Experiments). In ref 27, Moreno et al. applied the TDPAC technique, using ${ }^{181} \mathrm{Hf}\left(\rightarrow{ }^{181} \mathrm{Ta}\right)$ as implanted probes, to study the crystallization process of initially amorphous $\mathrm{Sn}-\mathrm{O}$ thin films to obtain the transparent and crystalline rutile $\mathrm{SnO}_{2}$ phase. ${ }^{181} \mathrm{Ta}$ was selected as PAC probe because the father isotope ${ }^{181} \mathrm{Hf}$ has the same nominal valence state as $\mathrm{Sn}, 4+$, not favoring oxygen vacancy trapping and hence allowing the study of a defect-free system. The success of the sample preparation was supported by two facts: (a) the good agreement between the hyperfine parameters obtained by CEMS at ${ }^{119} \mathrm{Sn}$ in 
undoped $\mathrm{Sn}-\mathrm{O}$ films in their final state, which underwent the same annealing process as the radioactive films, and the reported reference values of crystalline $\mathrm{SnO}_{2}$ phase in bulk; ${ }^{45}$ and (b) the EFG at ${ }^{181} \mathrm{Ta}$ in the $\mathrm{Sn}-\mathrm{O}$ films in their final state is almost coincident with the EFG at ${ }^{181} \mathrm{Ta}$ implanted in the single crystalline phase of transparent $\mathrm{SnO}_{2}$ thin films. ${ }^{26}$ This fact also showed that the presence of the impurity does not affect the overall crystallization process of the films.

On the other hand, the single and monochromatic hyperfine interaction found in both TDPAC experiments ${ }^{26,27}$ indicated also that all of the probes have the same local environment, but this fact does not confirm that the probes were located at the cationic sites nor that they could not have trapped a point defect. Now, from the excellent agreement between the experimental EFGs and the FP-APW+lo predictions, we can conclude that $100 \%$ of the ${ }^{181} \mathrm{Ta}$ impurities were located at defect-free substitutional $\mathrm{Sn}$ sites of crystalline $\mathrm{SnO}_{2}$, confirming the previous interpretations.

Nevertheless, in ref 27, the authors assumed that the inclusion of the Ta impurity produces enlargements in the TaONN bonds, instead of the contractions predicted by our FPAPW+lo calculations. That relaxation was proposed to reproduce the experimental $\eta$ parameter using simple PCM predictions. ${ }^{54}$ In this case, using an enlargement of $2.2 \%$ in the $\mathrm{Ta}-\mathrm{O} 2$ bond, the authors could reproduce an $\eta$ value of 0.7 , instead of 0.20 that is obtained by PCM using the $\mathrm{SnO}_{2}$ rutile structure.

Finally, in ref 27, the experimental results showed that the EFG does not change with temperature (in the range 300$1373 \mathrm{~K})$. To explain this, the authors suggested that the constancy of the EFG was due to the s character ( $S n$ states) of the conduction band, because the spherical symmetry of an ionized electron would not contribute to the EFG. At present, we know that a change in the EFG at a certain nucleus is related to a modification of the charge distribution in the close neighborhood of the probe-nucleus (i.e., in our calculations, within the muffin-tin radius of this atom). As shown in section III.B.2, only $2 \%$ of the charge of the impurity level at the edge of the conduction band is localized at the Ta atom (Ta-d states), while $98 \%$ belongs to O-2p and Sn-5s states. The large delocalization of this donor level can be also seen in the charge density plots of Figure 9. The potential removal of this little amount of charge from $\mathrm{Ta}$ at higher temperatures is compatible with a very small modification in the EFG (see Table 6) that cannot be resolved in a TDPAC experiment.

Let us now discuss the charge state of the Ta impurity in the case of real samples such as the Ta-doped $\mathrm{SnO}_{2}$ thin films used in TDPAC experiments ${ }^{27}$ and in resistivity and Hall effect measurements. ${ }^{6}$ Sometimes this charge state can be unambiguously determined through the comparison of the experimental EFG tensor with its ab initio prediction. ${ }^{55,56}$ For $\mathrm{Ta}$ concentrations equal to or less (e.g., ppm) than $4 \%$, the predicted EFG values in the APW+lo calculations for $\mathrm{SnO}_{2}: \mathrm{Ta}^{0}$ and $\mathrm{SnO}_{2}: \mathrm{Ta}^{+}$are almost coincident, and in excellent agreement with the experimental values of $V_{33}$ and $\eta$ coming from ${ }^{181} \mathrm{Ta}$ TDPAC experiments in ${ }^{181} \mathrm{Hf}$-implanted $\mathrm{SnO}_{2}$ thin films (see Table 6). Hence, for a diluted impurity-host system with substitutional $\mathrm{Ta}$ ions at $\mathrm{Sn}$ sites, the charge state of the impurities cannot be determined in this way.

The electrical properties of Ta-doped $\mathrm{SnO}_{2}$ thin films were determined through resistivity and Hall effect measurements for different $\mathrm{Ta}$ concentrations $(0-7 \%$ cation dilutions) by different authors. ${ }^{4-8}$ A clear semiconducting-metal transition was observed in the films when the Ta doping increases from $0.04 \%$ to $4 \%$, showing the lower resistivity $\left(1.1 \times 10^{-4} \Omega \mathrm{cm}\right)$ at $5 \%,{ }^{6}$ which is comparable to the lowest resistivity value of ITO thin films $\left(1 \times 10^{-4} \Omega \mathrm{cm}\right)$. The Ta dilution in our calculations is $4.2 \%$; therefore, in this case the system should have a metallic behavior and an optimal conductivity. When we study the electronic structure of a system doped with donor impurities, the impurity states could be localized either in the band gap as individual impurity levels near the conduction band or inside this band. In the first case, the localized single states can donate electrons by thermal promotion or optical transitions to the conduction band and the system will be semiconducting. In the second case, for high enough impurity concentrations, the impurity levels merge into an impurity band in the conduction band and it will have a metallic-like behavior (i.e, a degenerate semiconductor). For a $4.2 \% \mathrm{Ta}$ dilution, the DOS presented in Figure 5 shows that, at least for the neutral cell, the system is metallic, in agreement with the temperature dependence of the resistivity and the charge carrier density observed in Ta-doped $\mathrm{SnO}_{2}$ thin films, ${ }^{4,6}$ giving us an idea about the charge state of the impurity.

To obtain the correct charge state of the impurity, we calculated the defect formation energy for $\mathrm{SnO}_{2}: \mathrm{Ta}^{0}$ and $\mathrm{SnO}_{2}: \mathrm{Ta}^{+}$. The calculation was performed following the formalism described in detail in refs 23 and 57. The chemical potentials of $\mathrm{Sn}\left(\mu_{\mathrm{Sn}}\right)$ and $\mathrm{O}\left(\mu_{\mathrm{O}}\right)$ depend on the studied compound, and their values are rigorously limited by the experimental conditions. The chemical potentials show the loss of stoichiometry of a system and depend on different parameters, such as the partial pressures and growth conditions of the material. The chemical potentials $\mu_{\mathrm{Sn}}$ and $\mu_{\mathrm{O}}$ in $\mathrm{SnO}_{2}$ have a well-defined variation range, and their values depend on the several phases formed by them. The maximum values for $\mathrm{Sn}$ and $\mathrm{O}$ are limited by its value in the tetragonal metallic crystal $\left(\mu_{\mathrm{Sn}}^{*}\right)$ and by its value in the $\mathrm{O}_{2}$ molecule $\left(\mu_{\mathrm{O}}^{*}\right)$, respectively. To determine the variation of the Ta chemical potential $\left(\mu_{\mathrm{Ta}}\right)$, we need to consider the different compounds that the impurity can form when it interacts with the oxide host. The upper limit of $\mu_{\mathrm{Ta}}$ will be imposed by the chemical potential of the bcc metallic crystal $\left(\mu_{\mathrm{Ta}}^{*}\right)$. The calculation of $\mu_{\mathrm{Ta}}$ was performed taking into account the most stable phase of tantalum oxide (i.e., $\mathrm{Ta}_{2} \mathrm{O}_{5}$ ) as the reference element. In the present case, and taking into account an oxygen-rich neighborhood around the impurity, we calculated the formation energy $E_{\mathrm{f}}$ for both charge states using

$$
\begin{gathered}
E_{\mathrm{f}}\left(\mathrm{SnO}_{2}: \mathrm{Ta}\right)^{q}=E\left(\mathrm{SnO}_{2}: \mathrm{Ta}\right)^{q}-E\left(\mathrm{SnO}_{2}\right) \\
+\mu_{\mathrm{Sn}}^{*}+\Delta_{\mathrm{f}} H^{\mathrm{SnO}_{2}}-\mu_{\mathrm{Ta}}^{*} \\
-\frac{1}{2} \Delta_{\mathrm{f}} H^{\mathrm{Ta}_{2} \mathrm{O}_{5}}+q\left(\varepsilon_{\mathrm{F}}+\varepsilon_{\mathrm{v}}^{\prime}\right)
\end{gathered}
$$

Here, $\Delta_{\mathrm{f}} H$ are the enthalpy formations, $\varepsilon_{\mathrm{F}}$ is the Fermi energy in the band gap relative to the top of the valence-band energy $\varepsilon_{\mathrm{v}}^{\prime}$, with $0 \leq \varepsilon_{\mathrm{F}} \leq \varepsilon_{\mathrm{g}}\left(\varepsilon_{\mathrm{g}}\right.$ is the band gap energy), and $\varepsilon_{\mathrm{v}}^{\prime}=\varepsilon_{\mathrm{v}}+$ $\delta_{q}$, where $\varepsilon_{\mathrm{v}}$ is the top of the valence band energy of the pure system and $\delta_{q}$ lines up the band structures of the bulk material with and without impurity.

The obtained results showed that the $\mathrm{SnO}_{2}: \mathrm{Ta}^{0}$ system is the most stable for all values of the Fermi energy $\left(\varepsilon_{\mathrm{F}}\right)$ in the band gap, as Figure 10 shows. Hence, in samples with $\mathrm{Ta}$ concentrations of about $4 \%$ and without defects such as other acceptor impurities, the Ta impurity will prefer the neutral state 


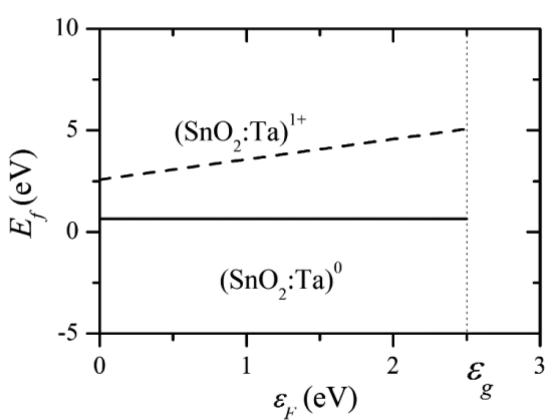

Figure 10. Defect formation energies $E_{\mathrm{f}}$ as a function of the Fermi energy level $\varepsilon_{\mathrm{F}}\left(0 \leq \varepsilon_{\mathrm{F}} \leq \varepsilon_{g}\right.$, where $\varepsilon_{\mathrm{g}}$ is the band gap energy). The black solid line corresponds to $\mathrm{SnO}_{2}: \mathrm{Ta}^{0}$ and the black dashed line to $\mathrm{SnO}_{2}: \mathrm{Ta}^{+}$.

and the system will be metallic, in agreement with the already mentioned experimental results of Tayosaki et al. ${ }^{6}$

It is worth mentioning here that the combination between TDPAC hyperfine characterizations and APW+lo predictions in Ta-doped $\mathrm{SnO}_{2}$ films with the impurity concentrations used in technological applications ${ }^{6-8}$ can be a valuable tool to give the local environment of the impurity in these cases. A TDPAC experiment in ${ }^{181} \mathrm{Ta}$-doped $\mathrm{SnO}_{2}$ with a $4 \%$ at. dilution could tell undoubtedly if the $\mathrm{Ta}$ impurities are at substitutional $\mathrm{Sn}$ sites or at interstitial ones, or forming $\mathrm{Ta}-\mathrm{O}$ clusters embedded in the host.

5. Comparison with Other Theoretical Calculations. Recently, in ref 28, Qin et al. reported ab initio DFT electronic structure calculations of Ta-doped $\mathrm{SnO}_{2}$ employing ultrasoft pseudopotentials within the CASTEP code. They predicted a negligible energy gap and a volume expansion of $0.4 \%$, attributed to the different ionic radii of $\mathrm{Sn}$ and Ta. As shown in section III.B.1, this expansion is originated in the very high Ta concentration used in their calculations (12.5 at. \% cationic dilution in a $2 \times 2 \times 1 \mathrm{SC}$ ), which cannot describe accurately neither the concentration of the doped samples in TDPAC experiments $(\mathrm{ppm})^{26,27}$ nor the samples used in resistivity and Hall effect measurements (with less than 4\%). ${ }^{6-8}$ This expansion is produced due to the repulsion of neighboring Ta atoms, which are separated only $3.4 \AA$ from each other along the $c$ axis before the expansion (see Figure 1). The Ta-ONN chemistry tries to shorten the $\mathrm{Ta}-\mathrm{O}$ bond-length, but, due to the fact that the $\mathrm{O} 1$ atoms are shared by neighboring Ta atoms along the $c$ axis, the $\mathrm{Ta}-\mathrm{O} 1$ distance expands around $0.6 \%$ in the $a-b$ plane toward the $\mathrm{Sn}$ atoms at the corners of the unit cell (Figure 1), which move expanding the cell volume. At the same time, the $\mathrm{Ta}-\mathrm{O} 2$ bond-length is shortened $1.5 \%$ along the [110] axis (see Figure 3a). For this very high Ta impurity concentration, the predicted EFGs for the unrelaxed and equilibrium structure for the neutral charge state are $V_{33}=$ $11.64 \times 10^{21} \mathrm{~V} / \mathrm{m}^{2}$ and $\eta=0.27$, and $V_{33}=4.56 \times 10^{21} \mathrm{~V} / \mathrm{m}^{2}$ and $\eta=0.26$, respectively, very different from those reported in Tables 5 and 6 . The differences between the EFG values for the unrelaxed structures at high and low Ta concentrations show that in the higher concentration case the Ta ions are interacting with each other.

\section{CONCLUSIONS}

Using the state-of-the-art FP-APW+lo ab initio AE method and considering different charge states of the impurity, we studied the local environment, the electronic structure, and the EFG tensor at Ta impurities placed at the cation sites of rutile $\mathrm{SnO}_{2}$.
As a starting point for the study of the doped system, we present APW+lo calculations of structural, electronic, and hyperfine properties in pure $\mathrm{SnO}_{2}$. These calculations show an excellent agreement with the experimental quadrupole splitting at ${ }^{119} \mathrm{Sn}$ in $\mathrm{SnO}_{2}$, using a recent determination of the quadrupole moment value for this isotope, and with XRD and XPS experiments.

In the doped system, the inclusion of the Ta impurity produces anisotropic contractions in the Ta-ONN bondlengths. The sense (inward or outward) and magnitude of the relaxations in Ta- and Cd-doped rutile $\mathrm{TiO}_{2}$ and $\mathrm{SnO}_{2}$ with respect to those of the pure oxides are proportional to the relative ionic radii between the impurity and the native cation of each oxide. The impurity tries to reconstruct the bond-length that $\mathrm{Ta}$ has in its most stable oxide $\mathrm{Ta}_{2} \mathrm{O}_{5}$, a general trend already observed in other ${ }^{181} \mathrm{Ta}$ - and ${ }^{111} \mathrm{Cd}$-doped metal oxides.

We show that Ta introduces an impurity donor level inside the bottom of the conduction band, which is partially filled in the neutral charge state, giving a metallic (degenerate semiconductor) behavior. The Ta contribution to this impurity level has mainly $d$ character, with a very small $\mathrm{p}$ contribution to the valence band. Only $0.22 \mathrm{e}^{-}$from the charge of the impurity level are localized at the Ta atoms.

After structural relaxations, only 0.02 electrons remained localized at the $\mathrm{Ta}$ atom. For both $\mathrm{SnO}_{2}: \mathrm{Ta}^{0}$ and $\mathrm{SnO}_{2}: \mathrm{Ta}^{+}$ systems, the $\mathrm{ab}$ initio calculations predict the same equilibrium structures and EFGs. The p contribution to $V_{33}$ is in all cases dominant. Nevertheless, the variation of $V_{33}$ as a function of the charge state of the impurity is dominated by the Ta-d contribution.

The Ta cationic dilution in our calculations is 4.2 at. \%, the same $\mathrm{Ta}$ concentration that gives the highest conductivity in the $\mathrm{SnO}_{2}$ thin films used in technological applications. Combining TDPAC experiments, ab initio EFG predictions, and defect formation energy calculations, we demonstrate that the implanted ${ }^{181} \mathrm{Ta}$ impurities with $\mathrm{ppm}$ concentration are located at defect-free substitutional $\mathrm{Sn}$ sites in the $\mathrm{SnO}_{2}$ thin films, and should present the metallic $\left(\mathrm{SnO}_{2}: \mathrm{Ta}^{0}\right)$ behavior of degenerate semiconductors. When the Ta impurity acts as an isolated impurity, the cell volume is not modified. A small volume expansion appears when the $\mathrm{Ta}$ concentration increases.

Finally, the accuracy obtained in the EFG predictions gives a benchmark for a future hyperfine experimental characterization of the local structure at the atomic level at impurity sites in Tadoped thin films having the optimal concentration for TCO applications. Also, the present study gives a complete knowledge of structural and electronic properties in the bulk system necessary for future theoretical investigations in $\mathrm{SnO}_{2}: \mathrm{Ta}$ surfaces and interphases, relevant in potential technological applications.

\section{AUTHOR INFORMATION}

\section{Corresponding Author}

*Tel.: 54-221-4230122/424720. E-mail: darriba@fisica.unlp. edu.ar.

\section{Notes}

The authors declare no competing financial interest.

\section{ACKNOWLEDGMENTS}

This work was partially supported by Consejo Nacional de Investigaciones Científicas y Técnicas (CONICET) under 
grant no. PIP0002, Argentina. This research made use of the HP-Parallel-Computing Bose Cluster, and the computational facilities of the Physics of Impurities in Condensed Matter group, at Instituto de Física La Plata and Departamento de Física.

\section{REFERENCES}

(1) Suzuki, K.; Mizuhashi, M. Structural, Electronical and Optical Properties of R.F.-Magnetron-Sputtered $\mathrm{SnO}_{2}: \mathrm{Sb}$ Film. Thin Solid Films 1982, 97, 119-127.

(2) Ma, J.; Hao, X.; Huang, S.; Huang, J.; Yang, Y.; Ma, H. Comparison of the Electrical and Optical Properties for $\mathrm{SnO}_{2}: \mathrm{Sb}$ Films Deposited on Polyimide and Glass Substrates. Appl. Surf. Sci. 2003, 214, 208-213.

(3) Lee, J. Effects of Oxygen Concentration on the Properties of Sputtered $\mathrm{SnO}_{2}: \mathrm{Sb}$ Films Deposited at Low Temperature. Thin Solid Films 2008, 516, 1386-1390.

(4) Lee, S. W.; Kim, Y.-W.; Chen, H. Electrical Properties of Tadoped $\mathrm{SnO}_{2}$ Thin Films Prepared by the Metal-Organic ChemicalVapor Deposition Method. Appl. Phys. Lett. 2001, 78, 350-352.

(5) Kim, Y.-W.; Lee, S. W.; Chen, H. Microstructural Evolution and Electrical Property of Ta-doped $\mathrm{SnO}_{2}$ Films Grown on $\mathrm{Al}_{2} \mathrm{O}_{3}$ (0001) by Metalorganic Chemical Vapor Deposition. Thin Solid Films 2002, 405, 256-262.

(6) Toyosaki, H.; Kawasaki, M.; Tokura, Y. Electrical Properties of Ta-doped $\mathrm{SnO}_{2}$ Thin Films Epitaxially Grown on $\mathrm{TiO}_{2}$ Substrate. Appl. Phys. Lett. 2008, 93, 132109.

(7) Nakao, S.; Yamada, N.; Hitosugi, T.; Hirose, Y.; Shimada, T.; Hasegawa, T. Fabrication of Highly Conductive Ta-doped $\mathrm{SnO}_{2}$ Polycrystalline Films on Glass using Seed-Layer Technique by Pulse Laser Deposition. Thin Solid Films 2010, 518, 3093-3096.

(8) Muto, Y.; Nakatomi, S.; Oka, N.; Iwabuchi, Y.; Kotsubo, H.; Shigesato, Y. High-Rate Deposition of Ta-doped $\mathrm{SnO}_{2}$ Films by Reactive Magnetron Sputtering using a Sn-Ta Metal-Sintered Target. Thin Solid Films 2012, 520, 3746-3750.

(9) Schatz, G.; Weidinger, A. Nuclear Condensed Matter Physics: Nuclear Methods and Applications; Wiley: Chichester, England, 1996.

(10) Kaufmann, E. N.; Vianden, R. J. The Electric Field Gradient in Noncubic Metals. Rev. Mod. Phys. 1979, 51, 161-214.

(11) Zhu, S.; Zhang, G.; Li, F.; Yuan, D. Proceedings of the 4th Joint International Conference on Hyperfine Interactions and International Symposium on Nuclear Quadrupole Interactions; Springer: Dordrecht, Netherlands, 2013.

(12) Lerf, A.; Butz, T. Nuclear Quadrupole Interaction and TimeResolved Perturbed $\gamma$ - $\gamma$-Angular Correlation Spectroscopy: Applications in Chemistry, Materials Science, and Biophysical Chemistry. New Analytical Methods. Angew. Chem., Int. Ed. Engl. 1987, 26, 110126.

(13) Klas, T.; Voigt, J.; Keppner, W.; Wesche, R.; Schatz, G. Characterization of Copper (100) Surfaces by Isolated Indium Probe Atoms via the Electric Field Gradient. Phys. Rev. Lett. 1986, 57, 10681071.

(14) Meersschaut, J.; L'abbe, C.; Rots, M.; Bader, S. D. Origin of Biquadratic Coupling in $\mathrm{Fe} / \mathrm{Cr}(100)$ Superlattices. Phys. Rev. Lett. 2001, 87, 107201.

(15) Ramallo López, J. M.; Rentería, M.; Miró, E. E.; Requejo, F. G.; Traverse, A. Structure of Extremely Nanosized and Confined In-O Species in Ordered Porous Materials. Phys. Rev. Lett. 2003, 91, 108304. (16) Lany, S.; Blaha, P.; Hamann, J.; Ostheimer, V.; Wolf, H.; Wichert, T.; Group, V. Acceptors in CdTe: $A b$ Initio Calculation of Lattice Relaxation and the Electric-Field Gradient. Phys. Rev. B 2000, 62, R2259-R2262.

(17) Errico, L. A.; Fabricius, G.; Rentería, M.; de la Presa, P.; Forker, M. Anisotropic Relaxations Introduced by $\mathrm{Cd}$ Impurities in Rutile $\mathrm{TiO}_{2}$ : First-Principles Calculations and Experimental Support. Phys. Rev. Lett. 2002, 89, 055503.

(18) Terrazos, L. A.; Petrilli, H. M.; Marszalek, M.; Saitovich, H.; Silva, P. R. J.; Blaha, P.; Schwarz, K. Electric Field Gradients at Ta in
Zr and Hf Inter-Metallic Compounds. Solid State Commun. 2002, 121, 525-529.

(19) Errico, L. A.; Fabricius, G.; Rentería, M. Metal Impurities in an Oxide: $A b$ Initio Study of Electronic and Structural Properties of $\mathrm{Cd}$ in Rutile $\mathrm{TiO}_{2}$. Phys. Rev. B 2003, 67, 144104 and references therein.

(20) Darriba, G. N.; Errico, L. A.; Eversheim, P. D.; Fabricius, G.; Rentería, M. First-Principles and Time-Differential $\gamma-\gamma$ PerturbedAngular-Correlation Spectroscopy Study of Structural and Electronic Properties of Ta-doped $\mathrm{TiO}_{2}$ Semiconductor. Phys. Rev. B 2009, 79, 115213.

(21) Forker, M.; de la Presa, P.; Hoffbauer, W.; Schalabach, S.; Bruns, M.; Szabó, D. B. Structure, Phase Transformations, and Defects of $\mathrm{HfO}_{2}$ and $\mathrm{ZrO}_{2}$ Nanoparticles Studied by ${ }^{181} \mathrm{Ta}$ and ${ }^{111} \mathrm{Cd}$ Perturbed Angular Correlations, ${ }^{1} \mathrm{H}$ Magic-Angle Spinning NMR, XPS, and XRay and Electron Diffraction. Phys. Rev. B 2008, 77, 54108.

(22) Jaeger, H.; Zacate, M. O. Defect and Diffusion Studied Using PAC Spectroscopy; Trans Tech Publications Ltd.: Zurich, Switzerland, 2011.

(23) Darriba, G. N.; Rentería, M.; Petrilli, H. M.; Assali, L. V. C. Site Localization of Cd Impurities in Sapphire. Phys. Rev. B 2012, 86, 075203.

(24) Hohenberg, P.; Kohn, W. Inhomogeneous Electron Gas. Phys. Rev. 1964, 136, B864-B871.

(25) Kohn, W.; Sham, L. J. Self-Consistent Equations Including Exchange and Correlation Effects. Phys. Rev. 1965, 140, A1133A1138.

(26) Moreno, M. S.; Desimoni, J.; Requejo, F. G.; Rentería, M.; Bibiloni, A. G.; Freitag, K. TDPAC Characterization of Tin Oxides Using ${ }^{181} \mathrm{Ta}$. Hyperfine Interact. 1990, 62, 353-358.

(27) Moreno, M. S.; Desimoni, J.; Bibiloni, A. G.; Rentería, M.; Massolo, C. P.; Freitag, K. Crystallization Study and Hyperfine Characterization of a Sn-O Thin Film With ${ }^{181}$ Ta. Phys. Rev. B 1991, 43, 10086-10092.

(28) Qin, G.; Zang, G.; Liu, S.; Fu, H.; Wen, J. Ab Initio Study on $\mathrm{Ta}: \mathrm{SnO}_{2}$. Adv. Mater. Res. 2011, 160-162, 574-578.

(29) Sjöstedt, E.; Nordström, L.; Singh, D. J. An Alternative Way of Linearizing the Augmented Plane-Wave Method. Solid State Commun. 2000, 114, 15-20.

(30) Madsen, G. K. H.; Blaha, P.; Schwarz, K.; Sjöstedt, E.; Nordström, L. Efficient Linearization of the Augmented Plane-Wave Method. Phys. Rev. B 2001, 64, 195134.

(31) Alonso, R. E.; Errico, L. A.; Peltzer y Blancá, E. L.; López-García, A.; Svane, A.; Christensen, N. E. Ab Initio Study of Structural and Electronic Properties and Hyperfine Interactions at the Ta Nucleus in Ta-doped Monoclinic $\mathrm{HfO}_{2}$. Phys. Rev. B 2008, 78, 165206.

(32) Decoster, S.; Cottenier, S.; Wahl, U.; Correia, J. G.; Vantomme, A. Lattice Location Study of Ion Implanted Sn and Sn-Related Defects in Ge. Phys. Rev. B 2010, 81, 155204.

(33) Taylor, M. A.; Alonso, R. E.; Errico, L. A.; López-García, A.; de la Presa, P.; Svane, A.; Christensen, N. E. Structural, Electronic, and Hyperfine Properties of Pure and Ta-Doped $m-\mathrm{ZrO}_{2}$. Phys. Rev. B 2012, 85, 155202.

(34) Bolzan, A. A.; Fong, C.; Kennedy, B. J.; Howard, C. J. Structural Studies of Rutile-Type Metal Dioxides. Acta Crystallogr., Sect. B 1997, 53, 373-380.

(35) Hazen, R. M.; Finger, L. W. Bulk Moduli and High-Pressure Crystal Structures of Rutile-Type Compounds. J. Phys. Chem. Solids 1981, 42, 143-151.

(36) Blaha, P.; Schwarz, K.; Madsen, G.; Kvasnicka, D.; Luitz, J. WIEN2k, An Augmented Plane Wave Plus Local Orbitals Program for Calculating Crystal Properties; Technical Universität Wien: Austria, 1999.

(37) Perdew, J. P.; Wang, Y. Accurate and Simple Analytic Representation of the Electron-Gas Correlation Energy. Phys. Rev. B 1992, 45, 13244-13249.

(38) Perdew, J. P.; Burke, K.; Ernzerhof, M. Generalized Gradient Approximation Made Simple. Phys. Rev. Lett. 1996, 77, 3865-3868.

(39) Wu, Z.; Cohen, R. E. More Accurate Generalized Gradient Approximation for Solids. Phys. Rev. B 2006, 73, 235116. 
(40) Schwarz, K.; Ambrosch-Draxl, C.; Blaha, P. Charge Distribution and Electric-Field Gradients in $\mathrm{YBa}_{2} \mathrm{Cu}_{3} \mathrm{O}_{7-x}$. Phys. Rev. B 1990, 42, 2051-2061.

(41) Blaha, P.; Schwarz, K.; Dederichs, P. H. First-Principles Calculation of the Electric-Field Gradient in HCP Metals. Phys. Rev. B 1988, 37, 2792-2796.

(42) Kohler, B.; Wilker, S.; Sheffler, M.; Couba, R.; Ambrosch-Draxl, C. Force Calculation and Atomic-Structure Optimization for the FullPotential Linearized Augmented Plane-Wave Code WIEN. Comput. Phys. Commun. 1996, 94, 31-48.

(43) Errico, L. A. Ab Initio FP-LAPW Study of the Semiconductors $\mathrm{SnO}$ and $\mathrm{SnO}_{2}$. Phys. B 2007, 389, 140-144.

(44) Barone, G.; Mastalerz, R.; Reiher, M.; Lindh, R. Nuclear Quadrupole Moment of ${ }^{119}$ Sn. J. Phys. Chem. A 2008, 112, 1666-1672.

(45) Collins, G. S.; Kachnowski, T.; Benczer-Koller, N.; Pasternak, M. Application of the Mössbauer Effect to the Characterization of an Amorphous Tin-Oxide System. Phys. Rev. B 1979, 19, 1369-1373.

(46) Dixit, H.; Sainz, R.; Cottenier, S.; Lamoen, D.; Partoens, B. Electronic Structure of Transparent Oxides with the Tran-Blaha Modified Becke-Johnson Potential. J. Phys.: Condens. Matter 2012, 24, 205503.

(47) Egdell, R. G.; Flavell, W. R.; Tavener, P. Antimony-Doped Tin(IV) Oxide: Surface Composition and Electronic Structure. J. Solid State Chem. 1984, 51, 345-354.

(48) Postnikov, A. V.; Entel, P.; Ordejón, P. $\mathrm{SnO}_{2}$ : Bulk and Surface Simulations by an $A b$ Initio Numerical Local Orbitals Method. Phase Transitions 2002, 75, 143-149.

(49) Nagasawa, M.; Shionoya, S. Exciton Structure in Optical Absorption of $\mathrm{SnO}_{2}$ Crystals. Phys. Lett. 1966, 22, 409-410.

(50) Agekyan, V. T. Spectroscopic Properties of Semiconductor Crystals with Direct Forbidden Energy Gap. Phys. Status Solidi A 1977, $43,11-42$.

(51) Koller, D.; Tran, F.; Blaha, P. Merits and Limits of the Modified Becke-Johnson Exchange Potential. Phys. Rev. B 2011, 83, 195134.

(52) Shannon, R. D. Revised Effective Ionic Radii and Systematic Studies of Interatomic Distances in Halides and Chaleogenides. Acta Crystallogr., Sect. A 1976, 32, 751-767.

(53) Darriba, G. N. Propiedades Estructurales y Electrónicas de Óxidos Semiconductores con Impurezas ${ }^{181} \mathrm{Ta} /{ }^{111} \mathrm{Cd}$ Diluidas. Estudio Experimental y de Primeros Principios. Ph.D. Thesis, Universidad Nacional de La Plata, La Plata, Argentina, March 2009. http://sedici. unlp.edu.ar/handle/10915/2619.

(54) Wiarda, D.; Uhrmacher, M.; Bartos, A.; Lieb, K. P. Electric Field Gradients at ${ }^{111} \mathrm{Cd}$ in Binary Oxides. J. Phys.: Condens. Matter 1993, 5, 4111-4124.

(55) Darriba, G. N.; Errico, L. A.; Muñoz, E. L.; Richard, D.; Eversheim, P. D.; Rentería, M. Electric-Field Gradients at Ta Donor Impurities in $\mathrm{Cr}_{2} \mathrm{O}_{3}(\mathrm{Ta})$ Semiconductor. Physica B 2009, 404, 27392741.

(56) Darriba, G. N.; Muñoz, E. L.; Eversheim, P. D.; Rentería, M. Experimental and $A b$ Initio Study of the Nuclear Quadrupole Interaction of ${ }^{181} \mathrm{Ta}$-Probes in an $\alpha-\mathrm{Fe}_{2} \mathrm{O}_{3}$ Single Crystal. Hyperfine Interact. 2010, 197, 207-212.

(57) Assali, L. V. C.; Machado, W. V. M.; Justo, J. F. Structural and Electronic Properties of $3 d$ Transition Metal Impurities in Silicon Carbide. Phys. Rev. B 2004, 69, 155212. 